\title{
Atypical Neuroleptic Malignant Syndrome: Pitfalls and Challenges in the Delirious Substance Abuser
}

\author{
Nirav N. Shah ${ }^{1}$, Kristin G. Fless ${ }^{1}$, Mikhail Litinski ${ }^{1}$, Fariborz Rezai ${ }^{1}$, Paul C. Yodice $^{1}$, \\ Henry Rosenberg ${ }^{2}$
}

${ }^{1}$ Department of Surgery and Critical Care Medicine, St. Barnabas Medical Center, Livingston, USA; ${ }^{2}$ Department of Medical Education and Clinical Research, St. Barnabas Medical Center, Livingston, USA.

Email:nirav@u.northwestern.edu

Received February $4^{\text {th }}, 2012$; revised February $26^{\text {th }}, 2012$; accepted March $4^{\text {th }}, 2012$

\begin{abstract}
Introduction: A rare and atypical form of Neuroleptic Malignant Syndrome (NMS) can be a deceptive and life threatening condition if not diagnosed properly in acute and critical care settings. Methods: The management of a patient presenting with atypical NMS without prominent rigidity, but with extensive rhabdomyolysis after the administration of haloperidol and ziprasidone is described in this report. Results: Prompt recognition of atypical features of NMS was managed by intensive care unit admission, supportive care and pharmacotherapy, leading to a complete resolution of the syndrome and a favorable outcome verified by laboratory findings. Conclusion: Early stages and atypical features of NMS may be variable in presentation and clinical course. The absence of muscle rigidity may not rule out NMS. A strong clinical suspicion based on clinical history is crucial for early diagnosis and treatment. Termination of dantrolene therapy may not be necessary during rhabdomyolysis and elevated aminotransferase levels.
\end{abstract}

Keywords: Atypical Neuroleptic Malignant Syndrome; Haloperidol; Ziprasidone; Dantrolene; Rhabdomyolysis; Creatinine Phosphokinase; Aminotransferase

\section{Introduction}

Neuroleptic malignant syndrome (NMS) is a rare, idiosyncratic, and potentially fatal adverse reaction associated with the use of antipsychotic medications. Since Delay et al. first described it in 1960, the syndrome has been associated with medications affecting central dopaminergic activity including antiemetics and sudden ces sation of dopaminergic anti-Parkinson's drugs [1-3]. While a definitive pathophysiological mechanism has not yet been elucidated, there is wide support linking NMS with dopamine-blockade of $\mathrm{D}_{2}$-receptors in central nervous system neurotransmission secondary to antipsychotic medications $[2,4,5]$. The incidence of NMS seems to have decreased from $3 \%$ to $0.01 \%-0.02 \%$ per year possibly due to increased awareness, changes in drug prescribing practices, the use of lower doses of dopamine blocking agents and atypical antipsychotics $[4,6]$. NMS, however, remains a significant source of morbidity and mortality for patients who receive antipsychotics [4]. Because NMS presents with a variety of signs and symptoms, its clinical diagnosis is accomplished by exclusion criteria based heavily on clinical history and clinician judgment $[2,7,8]$. Clinically it is characterized by motor symptoms, altered mental status, hyperthermia, and autonomic instability. While various diagnostic criteria are currently in use, a recent international consensus study has released a revised diagnostic criteria; however, they require further validation prior to clinical application [9].

The risk factors most consistently identified with precipitating NMS are prominent psychomotor agitation and incrementally higher doses of one or more parenterally administered neuroleptics [2,10]. The use of atypical antipsychotics may be associated with: a decreased risk, decreased mortality, and atypical features of NMS [4-6, 10,11], which has been challenged [12].

In this paper, we describe the case of a patient with a history of substance abuse, who presented with NMS atypically in regard to both a lack of muscular rigidity and an unusual course of the syndrome.

\section{Case Report}

A 41-year-old male with a past medical history of substance abuse, asthma, insomnia, and depression was admitted to the St. Barnabas Medical Center emergency department for evaluation of altered mental status which was noticed by his family overnight. He was found walking about his home confused, uttering nonsensical 
phrases and speaking to individuals who were not present, and was brought to the hospital by his parents.

A CT-scan of the head/brain without contrast was completed and showed no acute intracranial pathology. Initial vital signs were: temperature 98.9F, pulse 109 beats/min, blood pressure (BP) 143/94, and respiratory rate 22 breaths $/$ min with $97 \%$ saturation on room air. Initial chemical and metabolic panels were within normal limits, as was the urinalysis. A urine drug screen was positive for opiates.

The patient was initially treated with lorazepam $1 \mathrm{mg}$ IV and observed at bedside for response to treatment, with re-evaluations every 1 to 2 hours. At the first reassessment, the patient was resting more comfortably but still exhibited signs of pressured speech and psychotic thoughts. He was given 3 subsequent doses of lorazepam $1 \mathrm{mg}$ IV during re-evaluations. Subsequently, his persistent agitation prompted a $5 \mathrm{mg}$ IM dose of haloperidol. A plan was made to transfer the patient to a dedicated psychiatric facility. However, it was not possible to transfer the patient to the facility at the time, and another $2 \mathrm{mg}$ of IV of lorazepam was given to the patient for sedation at the request of the receiving institution.

After administration of haloperidol, the patient's status was unchanged for 2 hours. His agitation increased and he pushed a security guard and $40 \mathrm{mg}$ of oral ziprasidone was administered. A subsequent dose of lorazepam $2 \mathrm{mg}$ IV was given and the patient was managed with 4-point restraints due to increasing levels of agitation and delirium. Over the next few hours, the patient was given repeated doses of IV lorazepam totaling $8 \mathrm{mg}$. The family decided they did not want the patient to be transferred to the psychiatric facility as per the earlier plan of care.

Re-evaluation of the patient showed pupillary dilation, increased agitation and delirium which were ascribed to possible anti-cholinergic effects, however, the patient was also diaphoretic. Poison control was contacted. They suggested continuation of sedation with benzodiazepines and supportive care. His condition continued to worsen, and he was deemed to have a high probability of imminent life threatening deterioration in condition and an intensive care consult was called.

A rectal temperature of $101.4 \mathrm{~F}$ was measured, and at $11 \mathrm{pm}$ the patient was evaluated by an ICU resident due to signs of hemodynamic instability (Figure 1). Fluids were increased, labs and blood cultures drawn and sent. Upon receipt of an elevated white blood cell (WBC) count, the ICU resident was notified, another IV line started and fluid boluses given along with IV antibiotics. Bromocriptine (2.5 mg every 8 hours) and acyclovir (900 mg every 8 hours) were ordered and the patient was started empirically on both medications in order to treat for possible NMS vs Herpes simplex virus encephalitis.

Upon admission to the ICU the patient was febrile (101.4F), tachypneic (23 breaths/min), tachycardic (126 beats/min), and had an elevated WBC count of $17.0 \times$ $10^{3} / \mu \mathrm{L}$. In addition, other lab abnormalities included elevated levels of: creatinine $1.32 \mathrm{mg} / \mathrm{dL}$, aspartate aminotransferase (AST) 141 units/L, lactic acid $6.1 \mathrm{mmol} / \mathrm{L}$, and creatine phosphokinase (CPK) 14,121 units/L. Urinalysis showed abnormalities in protein $100 \mathrm{mg} / \mathrm{dL}$, trace leukocyte esterase, small amounts of ketones, large amounts of blood, WBC $33 / \mu \mathrm{L}, \mathrm{RBC} 345 / \mu \mathrm{L}$, and hyaline casts. Within 5 hours, his AST had climbed to 379

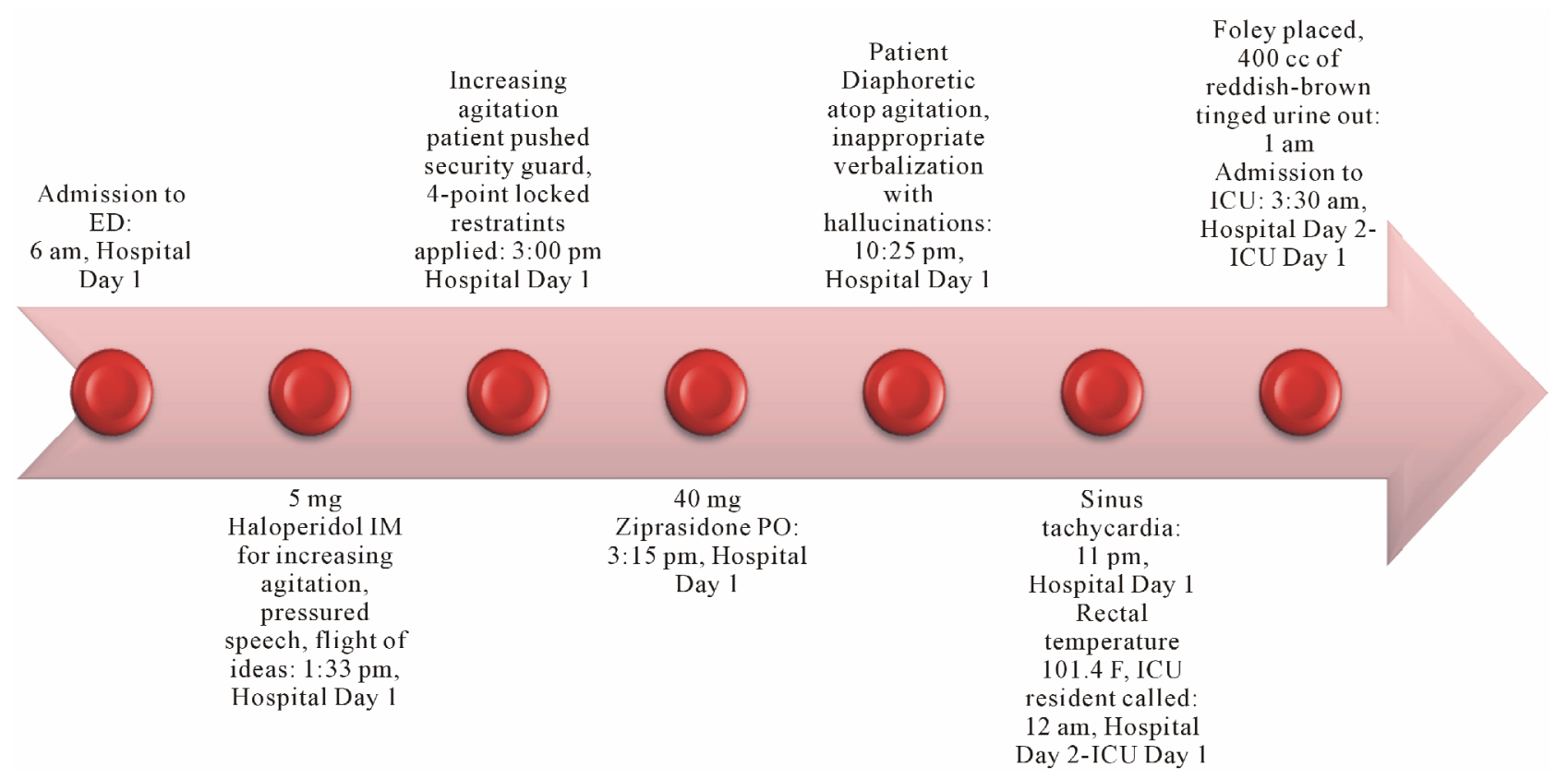

Figure 1. Timeline of important events leading up to ICU Admission with NMS. 
units/L, alanine aminotransferase (ALT) 53 units/L, and CPK 40,005. WBC count was still elevated but decreased to $12.8 \times 10^{3} / \mu \mathrm{L}$, and lactic acid down to $3.0 \mathrm{mmol} / \mathrm{L}$. A blood toxicity panel was ordered and sent out at this time to rule out phencyclidine (PCP), MDMA (ecstasy), and other stimulants. The blood panel subsequently came back negative. Within another 5 hours, CPK had risen to 84,340 units/L and dantrolene was ordered. Dantrolene was started in the evening of ICU day 1 (hospital day 2) at a rate of $235 \mathrm{mg}(2.5 \mathrm{mg} / \mathrm{kg}$, our patient weighed 95 $\mathrm{kg}$ ) over one hour with a repeat dose given six hours later. Further doses of dantrolene were held due to a sharp rise in liver enzymes (AST 680, ALT 137) but bromocriptine was continued at $2.5 \mathrm{mg}$ every 8 hours. An electroencephalogram study done later that day showed no abnormalities.

The following day (ICU day 2, hospital day 3), acyclovir was discontinued after an MRI of the head ruled out HSV encephalitis. CPK continued to drop steadily along with liver enzymes over the next two days as the patient received supportive care and bro-mocriptine.

He was transferred to a general medical floor on ICU day 4 (hospital day 5) and discharged to his home the next day with a plan to follow up with psychiatry as an outpatient. Prior to his departure, it was discovered that the patient had been abusing inhalants (e.g., computer dust remover cans) and had suffered from previous episodes of confusion and delirium similar to his initial presentation to the ED.

\section{Discussion}

To the best of our knowledge, NMS involving ziprasidone has been described fewer than 10 times in the literature to date [12]. This may be the first case described involving a combination of both haloperidol and ziprasidone to treat delirium and agitation acutely leading to NMS. Furthermore, the present case may be unusual in the rapidity of NMS onset and subsequent recovery in our patient. NMS onset typically evolves insidiously over a period of days, with $66 \%$ of cases in one review occurring within the first week and virtually all cases within 30 days [5]. Our patient developed full blown NMS within 7 hours of his last dose of ziprasidone and what appear to be prodromal signs almost immediately after the first dose of antipsychotic medication. The recovery time in uncomplicated NMS cases is typically between seven to 10 days [2]. Our patient was well enough to be transferred to a general medical floor well on his way to recovery three to four days after onset. While it is known that higher doses and the use of two or more neuroleptics are risk factors for precipitating NMS [2], it is not known what effect this may have on the onset and course of the syndrome.
Many patients with delirium are treated with antipsychotics, which continue to be recommended in professional guidelines as the gold-standard treatment [6]. However, in over $80 \%$ of cases, altered mental status presenting as confusion, catatonia, delirium or agitation is the first sign of NMS before systemic signs appear [4]. This poses a diagnostic dilemma, especially in the critical care setting where concomitant use of sedatives may obscure the sentinel signs of NMS, leading to unusual presentations which may delay timely diagnosis $[6,13]$. Adherence to a stricter set of criteria such as the DSM-IV may further compound the problem. Atypical antipsychotics have been described to cause NMS "atypically" in that they lack the classic rigidity and motor symptoms commonly seen with typical antipsychotics $[5,7,8]$, a finding consistent with our patient's presentation. Some studies have suggested that this may be due to the antagonistic effects atypical antipsychotics have on receptors other than $\mathrm{D}_{2}-\mathrm{R}$, namely $5 \mathrm{HT}_{2}-\mathrm{R}[5,8]$. This may be reflective of an alternative biochemical mechanism of drug action, which, may ultimately reflect on the varied clinical pictures of NMS; similar in concept to the mechanisms responsible for the antiemetic and gastroprokinetic effects of metoclopromide [14-16]. The DSMIV criteria requires that both severe muscle rigidity and elevated temperature must be present after recent administration of an antipsychotic as well as two associated signs, symptoms, or laboratory findings that are not better accounted for by other causes; this may lead clinicians to an alternate diagnosis $[2,4]$. It, however, seems clear that the principles of pharmacodynamics and drug antagonism need to be considered for the management of the patient.

While dantrolene therapy was terminated in this case due to concerns of hepatotoxicity, this may not have been necessary as the literature describes a correlation between elevated CPK and serum aminotransferase secondary to rhabdomyolysis $[17,18]$. Additionally, the incidence of acute liver injury with hepatic dysfunction is present in only $25 \%$ of patients with non-traumatic rhabdomyolysis and is reversible [17]. Furthermore, our patient's CPK and aminotranssferase levels followed a pattern described in a recent study [19] suggestive of a muscle isoenzyme source for the elevated aminotransferases seen (Table 1). Indeed, dantrolene therapy seemed to be very effective at resolving signs of NMS in this case.

Prior to discharge, we learned that our patient was an abuser of inhalants, and the question of inhalant abuse as a potential cause for his rhabdomyolysis was raised. While an association between toluene in many commonly abused inhalants and rhabdomyolysis has been described in the literature [20,21], we find it unlikely to be the etiology of our patient's elevated CPK levels 
Table 1. Pertinent laboratory values from arrival in ED through admission and stay in ICU.

\begin{tabular}{cccccccccc}
\hline $\begin{array}{c}\text { Pertinent } \\
\text { Labs }\end{array}$ & $\begin{array}{c}\text { ED Admit } \\
\text { Labs }\end{array}$ & $\begin{array}{c}\text { ICU Day 1 } \\
\text { Midnight }\end{array}$ & $\begin{array}{c}\text { ICU Day 1 } \\
\text { AM Labs }\end{array}$ & $\begin{array}{c}\text { ICU Day 1 } \\
\text { Noon Labs }\end{array}$ & $\begin{array}{c}\text { ICU Day 1 } \\
\text { PM Labs }\end{array}$ & $\begin{array}{c}\text { ICU Day 2 } \\
\text { Midnight }\end{array}$ & $\begin{array}{c}\text { ICU Day 2 } \\
\text { AM Labs }\end{array}$ & $\begin{array}{c}\text { ICU Day 3 } \\
\text { AM Labs }\end{array}$ & $\begin{array}{c}\text { ICU Day 4 } \\
\text { AM Labs }\end{array}$ \\
\hline WBC & 8.7 & $\mathbf{1 7 . 0}$ & $\mathbf{1 2 . 8}$ & - & - & - & 9.3 & 8.3 & 9.0 \\
BUN & 20 & 22 & 20 & 18 & 13 & 10 & 10 & 10 & 9 \\
Creatinine & 1.2 & $\mathbf{1 . 3 2}$ & 1.16 & 1.03 & 0.83 & 0.80 & 0.77 & 0.72 & 0.73 \\
CPK & - & $\mathbf{1 4 , 1 2 1}$ & $\mathbf{4 0 , 0 0 5}$ & $\mathbf{8 4 , 3 4 0}$ & $\mathbf{6 8 , 4 9 0}$ & $\mathbf{4 1 , 6 1 0}$ & $\mathbf{3 1 , 2 6 0}$ & $\mathbf{1 4 , 4 4 3}$ & $\mathbf{9 2 6 8}$ \\
AST & 18 & $\mathbf{1 4 1}$ & $\mathbf{3 7 9}$ & - & - & $\mathbf{6 8 0}$ & $\mathbf{5 6 0}$ & $\mathbf{4 1 2}$ & $\mathbf{3 5 4}$ \\
ALT & 14 & 29 & $\mathbf{5 3}$ & - & - & $\mathbf{1 3 7}$ & $\mathbf{1 3 2}$ & $\mathbf{1 3 0}$ & $\mathbf{1 4 4}$ \\
Anion Gap & 15 & $\mathbf{2 2}$ & 15 & 16 & 13 & 10 & 8 & 9 & 12 \\
\hline
\end{tabular}

given his clinical history, time course, and laboratory values.

\section{Conclusion}

Although NMS in its full-blown classic form is unmistakable, the early stages, including atypical forms of the disease may be variable in presentation and clinical course. The present case of NMS is significant in that it shows an atypical early absence of muscle rigidity, despite extensive rhabdomyolysis and elevated CPK. Hence, an adherence to strict diagnostic criteria may lead to alternate and/or delayed diagnoses with poor outcomes.

\section{REFERENCES}

[1] J. Delay, P. Pichot, T. Lemperiere, B. Elissalde and F. Peigne, "A Non-Phenothiazine and Non-Reserpine Major Neuroleptic, Haloperidol, in the Treatment of Psychoses," Annales Medico-Psychologiques, Vol. 118, No. 1, 1960, pp. 145-152.

[2] M. J. Bhanushali and P. J. Tuite, "The Evaluation and Management of Patients with Neuroleptic Malignant Syndrome," Neurologic Clinics, Vol. 22, No. 2, 2004, pp. 389-411. doi:10.1016/i.ncl.2003.12.006

[3] K. J. McAllen and D. R. Schwartz, "Adverse Drug Reactions Resulting in Hyperthermia in the Intensive Care Unit," Critical Care Medicine, Vol. 38, 2010, pp. S244S252. doi:10.1097/CCM.0b013e3181dda0d4

[4] J. R. Strawn, P. E. Keck Jr. and S. N. Caroff, "Neuroleptic Malignant Syndrome," The American Journal of Psychiatry, Vol. 164, No. 6, 2007, pp. 870-876. doi:10.1176/appi.ajp.164.6.870

[5] M. C. Borovicka, L. C. Bond and K. M. Gaughan, “Ziprasidone- and Lithium-Induced Neuroleptic Malignant Syndrome," The Annals of Pharmacotherapy, Vol. 40, No. 1, 2006, pp. 139-142. doi:10.1345/aph.1G470

[6] D. P. Seitz and S. S. Gill, "Neuroleptic Malignant Syndrome Complicating Antipsychotic Treatment of Delirium or Agitation in Medical and Surgical Patients: Case Reports and a Review of the Literature," Psychosomatics, Vol. 50, No. 1, 2009, pp. 8-15.

\section{doi:10.1176/appi.psy.50.1.8}

[7] M. Paul, S. G. Michael, S. John and R. J. Lenox, "An Atypical Presentation of Neuroleptic Malignant Syndrome: Diagnostic Dilemma in a Critical Care Setting," Respiratory Care, Vol. 57, No. 2, 2011, pp. 315-317.

[8] L. S. Picard, S. Lindsay, J. R. Strawn, R. M. Kaneria, N. C. Patel and P. E. Keck Jr., "Atypical Neuroleptic Malignant Syndrome: Diagnostic Controversies and Considerations," Pharmacotherapy, Vol. 28, 2008, pp. 530-535. doi:10.1592/phco.28.4.530

[9] R. J. Gurrera, S. N. Caroff, A. Cohen, et al., “An International Consensus Study of Neuroleptic Malignant Syndrome Diagnostic Criteria Using the Delphi Method," The Journal of Clinical Psychiatry, Vol. 72, No. 9, 2011, pp. 1222-1228. doi:10.4088/JCP.10m06438

[10] V. P. Kontaxakis, B. J. Havaki-Kontaxaki, S. S. Stamouli and G. N. Christodoulou, "Toxic Interaction between Risperidone and Clozapine: A Case Report," Progress in Neuro-Psychopharmacology and Biological Psychiatry, Vol. 26, No. 2, 2002, pp. 407-409. doi:10.1016/S0278-5846(01)00257-3

[11] M. Nakamura, H. Yasunaga, H. Miyata, T. Shimada, H. Horiguchi and S. Matsuda, "Mortality of Neuroleptic Malignant Syndrome Induced by Typical and Atypical Antipsychotic Drugs: A Propensity-Matched Analysis from the Japanese Diagnosis Procedure Combination Database," The Journal of Clinical Psychiatry, 2011.

[12] J. N. Trollor, X. Chen and P. S. Sachdev, "Neuroleptic Malignant Syndrome Associated with Atypical Antipsychotic Drugs," CNS Drugs, Vol. 23, No. 6, 2009, pp. 477492. doi:10.2165/00023210-200923060-00003

[13] S. N. Caroff, H. Rosenberg, S. C. Mann, E. C. Campbell and K. A. Sullivan, "Neuroleptic Malignant Syndrome in the Critical Care Unit," Indian Journal of Critical Care Medicine, Vol. 10, No. 1, 2002, pp. 50-52.

[14] L. Justin-Besancon and C. Laville, "Antiemetic Action of Metoclopramide with Respect to Apomorphine and Hydergine," Comptes Rendus des Seances de la Societe de Biologie et de Ses Filiales, Vol. 158, 1964, pp. 723-727.

[15] H. P. Rang, M. M. Dale, J. M. Ritter and P. K. Moore, "Pharmacology," 5th Edition, Churchill Livingstone, Edinburgh, 2003.

[16] M. Tonini, S. M. Candura, E. Messori and C. A. Rizzi, 
"Therapeutic Potential of Drugs with Mixed 5-HT4 Agonist/5-HT3 Antagonist Action in the Control of Emesis," Pharmacological Research: The Official Journal of the Italian Pharmacological Society, Vol. 31, No. 5, 1995, pp. 257-260.

[17] M. Akmal and S. G. Massry, "Reversible Hepatic Dysfunction Associated with Rhabdomyolysis," American Journal of Nephrology, Vol. 10, 1990, pp. 49-52. doi:10.1159/000168053

[18] A. L. Huerta-Alardin, J. Varon and P. E. Marik, "Benchto-Bedside Review: Rhabdomyolysis-An Overview for Clinicians," Critical Care, Vol. 9, 2005, pp. 158-169. doi:10.1186/cc2978

[19] K. Weibrecht, M. Dayno, C. Darling and S. B. Bird,
"Liver Aminotransferases Are Elevated with Rhabdomyolysis in the Absence of Significant Liver Injury," Journal of Medical Toxicology, Vol. 6, No. 3, 2010, pp. 294-300. doi:10.1007/s13181-010-0075-9

[20] G. C. Karmakar and R. Roxburgh, "Rhabdomyolysis in a Glue Sniffer," The New Zealand Medical Journal, Vol. 121, No. 1271, 2008, pp. 70-71.

[21] K. C. Kao, Y. H. Tsai, M. C. Lin, C. C. Huang, C. Y. Tsao and Y. C. Chen, "Hypokalemic Muscular Paralysis Causing Acute Respiratory Failure due to Rhabdomyolysis with Renal Tubular Acidosis in a Chronic Glue Sniffer," Journal of Clinical Toxicology, Vol. 38, 2000, pp. 679-681. doi:10.1081/CLT-100102021 